\title{
An Alinement Interferometer
}

\author{
J. B. Saunders
}

(July 25, 1963)

\begin{abstract}
This paper describes a diverging-beam type of alinement interferometer that permits the use of large apertures, provides ruggedness, high sensitivity, and is relatively compact. Since this interferometer is fully compensated, white light can be used.

The narrowness of the zero order fringe, relative to separation of fringes, in such a well compensated system permits settings to better than one twentieth of a fringe width. This corresponds to a lateral displacement of the light source of less than $1.5 \mathrm{~mm}$ at a distance of one mile for a one-inch aperture instrument and to less than $0.15 \mathrm{~mm}$ for a 10 -inch aperture instrument.

The increase in dmands for higher accuracy in metrology includes that of pointing. The alinement interferometer described here is a precision device that is rugged and relatively easy to apply. Its performance, because of its high sensitivity, is severally affected by the homogeneity of the atmosphere or medium through which the light passes. However, this effect is also greatly reduced by the proximity of any two interfering rays of light.
\end{abstract}

There appears to be a growing need for an accurate method of alining objects over distances of several miles. The diffraction methods, described by Van $\mathrm{Heel}^{1}$ and Dyson ${ }^{2}$, appear to be inadequate for such distances. Other methods such as the axicon ${ }^{3}$ and the telescope also have limited accuracy. The alinement interferometer described here is believed to be practical and more sensitive than previously described methods.

An arrangement of optical elements that form a precision alinement interferometer is shown in figure 1. It consists of two double-image prisms ${ }^{4}, P_{1}$ and $P_{2}$, a beam divider, $B$, a lens, $L$, a source, $S$, and two pairs of quarter-wave plates, $C_{1}$ and $C_{2}$. Figure $2 \mathrm{~A}$ shows the course of the light from $B$ to the observer located either at $F^{\prime}{ }_{1}$ or $F^{\prime \prime}{ }_{1}$ for measurements in the plane of figure 1 , and figure $2 \mathrm{~B}$ shows the same for measurement in the plane perpendicular to that of figure 1.

The alinement problem may consist of determining the position of the source, $S$, located a distance, $d$, from the optic axis of $L$, the precise adjustment of $S$

1 Van Heel, A.C.S., Progress in optics 1, 291 (1961).

2 Dyson, J., Opties in metrology, p. 169 (Pergamon Press, Pol. Mollet Ed., 1960

${ }^{3}$ MeLeod, John H., The axicon., JOSA 44, 592 (1954).

4 J. B. Saunders, Construction of a Kösters double-image prism; J. Res. NBS 58, 21-26 (1957). on the axis of $L$, or the alinement of several sources along the axis of $L$. Obviously, if the problem is to determine the position of $S$ relative to one plane only (the plane of fig. 1, for instance), then $P_{2}$ and $B$ may be eliminated.

The optical sensitivity of this interferometer is proportional to the aperture. If the aperture of the prism is sufficient, the lens may be eliminated and the entrance tace of the prism may be plane or spherical. A plane face prism, used without the aperture-increasing lens $L$, requires a collector lens as shown in figure 3. The $2 \mathrm{~d}$ prism and beam divider are not shown in figure 3. They are, however, necessary for alinement in the plane perpendicular to this figure.

This prism is difficult to make in large sizes. Small prisms are relatively easy to construct and may be used with a lens to increase the aperture of the interferometer. Aperture sizes are limited only by the difficulty of producing large objectives (lenses or mirrors). A mirror objective is as easily used as a refractor and is free from chromatic aberration.

The equation of sensitivity is obtained from figure 4 , in which the aperture is represented by the aperture of a lens, $L$. Consider two rays of light that emerge from the lens at points $A_{1}$ and $A_{2}$ that are equally

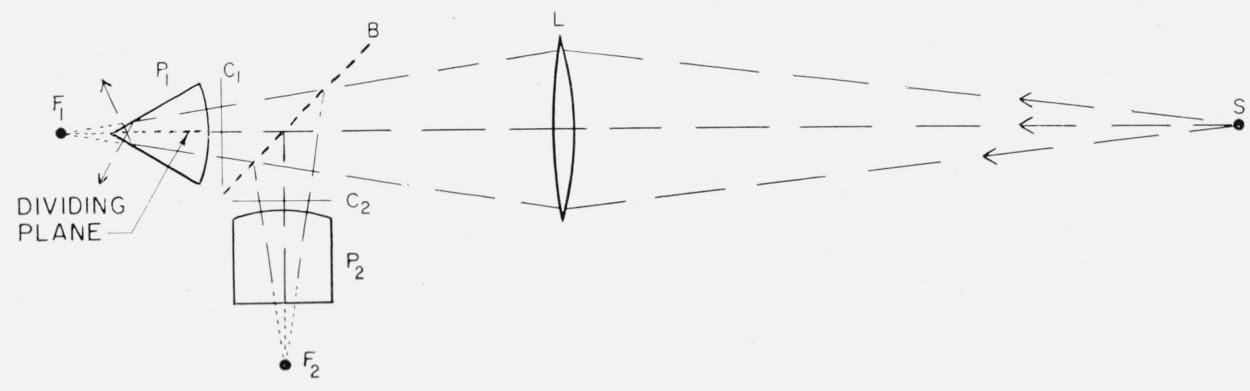

Figure 1. Plane view of optical elements.

The dividing plane of the double-image prism is a s?mi-reflecting optical film. It serves as the beam combiner in this interferometer. 


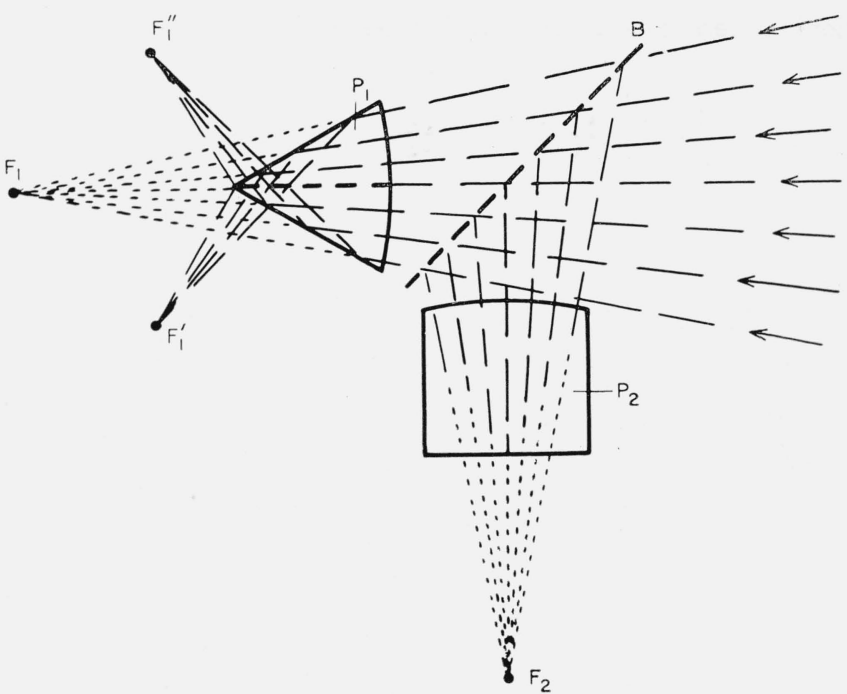

A

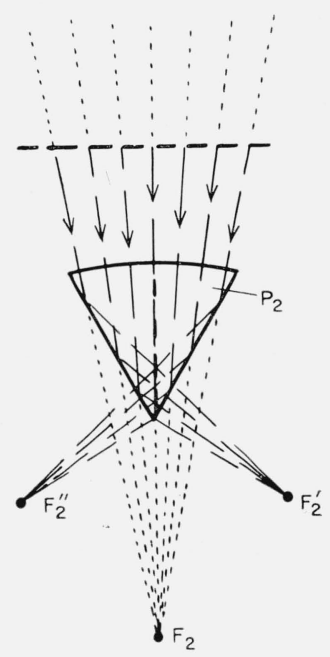

B

FiguRE 2. Figure $2 A$ represents the path of the light in the plane of figure 1 . Figure $2 B$ represents the same in a plane perpendicular to the plane of figure 1.

The intersection of these two planes represent the straight line of interest.

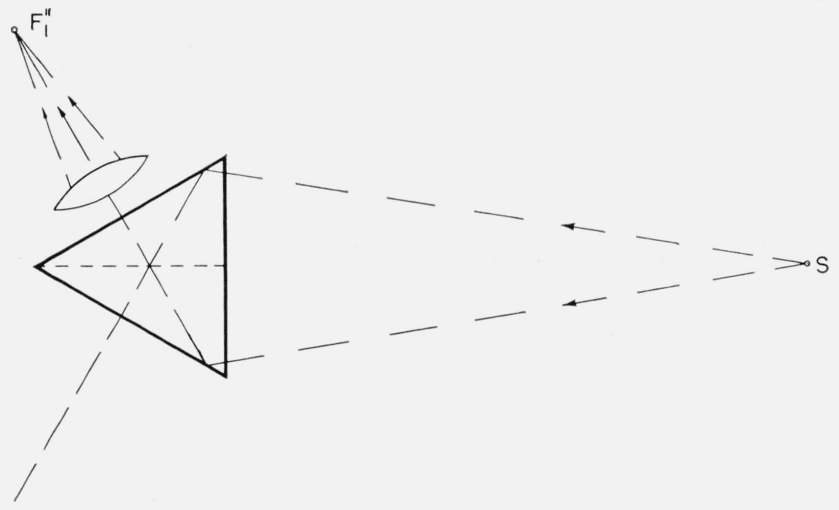

Figure 3. Optics of an alinement interferometer.

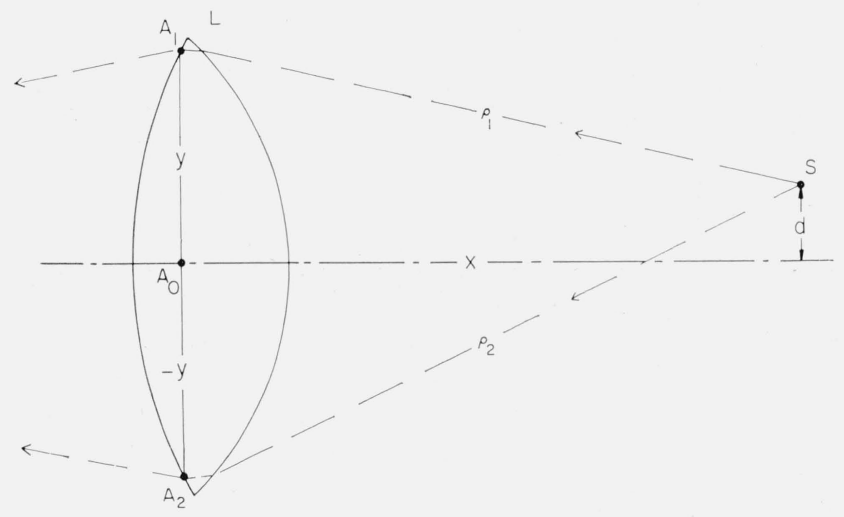

Figure 4. Rays of light, $\rho_{1}$ and $\rho_{2}$, are combined in the prism. Consequently, points $\mathrm{A}_{1}$ and $\mathrm{A}_{2}$ appear to coincide at the reference points, indicated in figures 5 and 6. distant $\left(y\right.$ and $-y$ ) from $A_{0}$, which lie in the extension of the dividing plane of prism $P_{1}$. The distance $X$ (approximating the distance from lens to source), shown in figure 4 , is measured from $A_{0}$. The plane of figure 4 is assumed to include the optic axis of $L$. Let $\rho_{1}$ and $\rho_{2}$ equal the optical paths from the source to the observer (at $F_{1}^{\prime \prime}$ or $F_{1}^{\prime \prime \prime}$, fig. 2 ) through $A_{1}$ and $A_{2}$, respectively. The optical path from $A_{1}$ to the observer is identical to that from $A_{2}$, because of symmetry in the prism. The observer sees images of $A_{1}$ and $A_{2}$ superimposed, as shown in figure 5. The observed order of interference, $N$, at this pair of points, multiplied by the wavelength of the light, $\lambda$, is equal to $\left(\rho_{1}-\rho_{2}\right)$. Since $X$ is assumed quite large relative to $y$ and $d$, we can ignore the refractive index of the lens and obtain,

$$
\begin{aligned}
& \rho_{1}^{2}=X^{2}+(y-d)^{2}, \\
& \rho_{2}^{2}=X^{2}+(y+d)^{2} .
\end{aligned}
$$

On subtracting eq (1) from (2), and espanding, we readily obtain

$$
\rho_{2}^{2}-\rho_{1}^{2}=\left(\rho_{2}-\rho_{1}\right)\left(\rho_{2}+\rho_{1}\right)=4 y d .
$$

On putting $\left(\rho_{2}+\rho_{1}\right)=2 X$ (which it approximates for large values of $X$ ) and solving for $d$ we obtain

$$
d=\frac{2 X \cdot N \lambda}{4 y}
$$

If $A$ equals the aperture of the interferometer and the reference point is chosen at or near the margin then $y=1 / 2 A$ and

$$
d=\frac{X N \lambda}{A}
$$




\section{allilis dillı dillı}

FIGURE 5. The appearance of interference fringes when the point source is: A, above the line; B, on the line; and $\mathrm{C}$, below the line of interest.

If the problem is to adjust $S$ on the axis, and the prism is adjusted to place the zero order of interference on the reference point where $d=0$, then the adjustment consists in moving $S$ perpendicular to the axis until $N$ equal zero. If white light is used with a reflecting objective (a parabolic mirror), the zero order of interference appears to have excellent contrast and an experienced observer can adjust the fringes to an accuracy of less than 0.05 fringe spacing. The corresponding error in $N$ is 0.05 and for $\lambda=5.0$ $\times 10^{-5} \mathrm{~cm}$ (the approximate effective value for white light sources), the corresponding error in $d$ is $X / A$ $\times 2.5 \times 10^{-6} \mathrm{~cm}$. This corresponds to an error in $d /$ (the angular position of $S)$ of $\frac{2.5}{A} \times 10^{-6}(A$ expre: sed in $\mathrm{cm}$ ). If $A$ equals 6 in. and $X$ equals 1 mils, the corresponding error in $d$ is approximately $0.3 \mathrm{~mm}$.

Two photomultiplier tubes placed in a differential arrangement can detect changes in the position of $S$ to much less than this. Obviously, this principle can be used to guide an astronomical telescope when mounted on a satellite (above the atmosphere of the eart's) to very high precision.

Visual observations are most easily made with a prism that has a "built-in-wedge" of convenient magnitude to produce interference fringes whose widths are most appropriate for precision of readings. Prisms may easily be adjusted to provide fringes that are neither too broad nor too narrow. When photoelectric recording is to be used for null settings the prism should be adjusted to have no built-in wedge. This permits the use of the entire beam for detection.

Figures $5 \mathrm{~A}, 5 \mathrm{~B}$, and $5 \mathrm{C}$ respectively show the appearance of the fringes in a built-in wedge interferometer of 4 in. aperture when $S$ is one second of arc above, on, and one second below, the dividing plane of the prism through which observation is made.

Because of differential polarization of light in the prism, unpolarized light cannot be used without properly oriented quarter-wave plates or a polarizer. Use of polarized light results in a loss of one-half the available light. The polarizer is used by placing. it either between the prism and observer or near the source. If a polarizer is adjusted to transmit light vibrating parallel to the dividing plane of the prism, and if the observed fringe pattern is represented by figure $6 \mathrm{~A}$, then the pattern will change to that of figure $6 \mathrm{~B}$ when the polarizer is rotated $90^{\circ}$ (light vibrating perpendicular to the dividing plane of the prism). Therefore, unpolarized

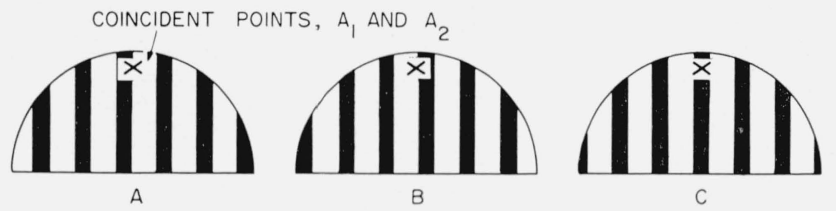

FIGURE 6. The appearance of interference fringes when: A, the light is polarized parallel to the dividing plane of figure 1; $\mathrm{B}$, the light is polarized perpendicular to the plane of figure 1; and $\mathrm{C}$, when quarter-wave plates are used instead of a polarizer.

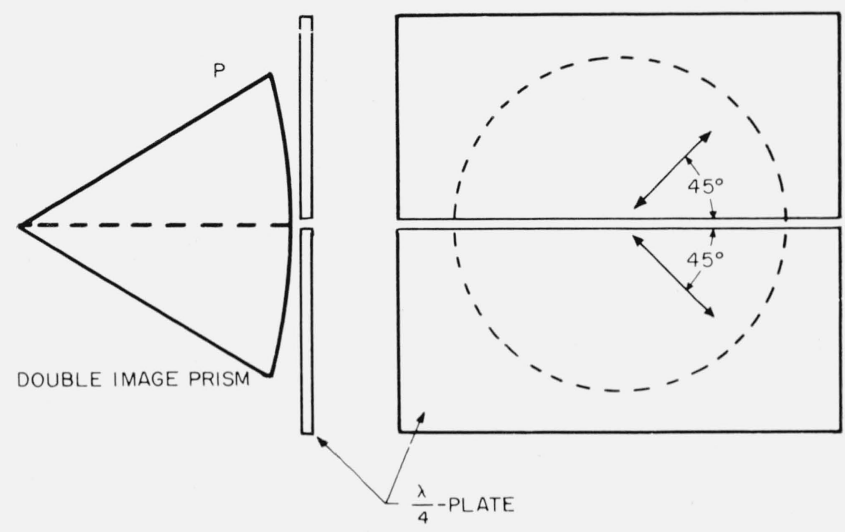

Figure 7. Arrangement of quarter-wave plates to give good contrast with unpolarized light.

light produces two sets of fringes with the maximum intensity areas of one set coinciding approximately, with the minimum intensity areas of the other set, thus eliminating contrast so that no fringes can be observed.

Dr. Charles J. Koester ${ }^{5}$ suggested and demonstrated that by cutting a quarter-wave sheet ${ }^{6}$ of mica at $45^{\circ}$ to its optic axis and mounting it in front of the prism, in the manner shown in figure 7 , the relative phase changes in the two polarized beams are practically equalized. The two sets of fringes produced by the two component beams (vibrating parallel and perpendicular to the dividing plane of the prism) will then coincide in phase. Good contrast is thereby obtained with little loss of light. The resultant fringe pattern (fig. 6C) indicates that the two sets of fringes, shown in figures $6 \mathrm{~A}$ and $6 \mathrm{~B}$, are shifted in opposite directions by an amount that places them in coincidence.

Tests made in the laboratory showed that the sensitivity of the instrument, obtained from eq (3), agreed within the limits of observations. The theoretical accuracy claimed above requires that the medium through which the light travels be quite homogeneous. An evacuated light tube would probably be required to obtain the accuracy indicated by eq (3) when measurements are made over long: distances.

${ }^{5}$ Working at NBS under a National Research Council Postdoctoral Research Associateship and now with American Optical Co., Southbridge, Mass.

6 Subsequent tests showed that the relative retardation is not critical for obtaining good contrast.

(Paper 67C4-141) 